killed by predators and parasites immediately, or by parasites within a period of three weeks, after the fumigation.

As no resistant mutants thus survived the treatment by the selective insecticide and the following attack by the beneficial insects, any possibility of a segregation of a more resistant race was precluded.

The search for further selective insecticides would therefore seem to be a promising approach to the extension of the usefulness of chemical control. More rapid progress could be achieved if the comparative physiology of the economically important insect groups were systematically explored.

${ }^{1}$ Melander, A. 1.., J. Econ. Ent., 7, 167 (1914).

${ }^{2}$ Quayle, H. J., Cal. Univ. J. Agric., 3, (8), 333, 358 (1916).

${ }^{3}$ Quayle, H. J., Hilgardia, 11 (5), 183 (1938).

- Woglum, R. S., J. Econ. Ent., 18, 593 (1925).

${ }^{5}$ Dickson, R. C., Hilgardia, 13 (9), 515 (1940).

- Hough, W. S., J. Econ. Ent. 21, 325 (1928).

${ }^{2}$ Hough, W. S., J. Agric. Res., 38, 245 (1929)

${ }^{8}$ Hough, W. S., J. Agric. Res., 48, 533 (1934).

' Knipling, E. F., J. Econ. Ent., 35, 63 (1942).

${ }^{10}$ Boyce, A. M., Persing, C. O., and Barnhart, C. S., J. Econ. Ent. 35,790 (1942).

${ }^{11}$ Smith, H. S., J. Econ. Ent., 34 (1), 1 (1941).

${ }^{12}$ Quayle, H. J., J. Econ. Ent, 36 (4), 493 (1943).

${ }^{13}$ Yust, H. R., Nelson, H. D., and Busbey, R. L., J. Econ. Ent., 36, (5), 744-749 (1943).

${ }^{14}$ Volterra, V., J. Conseil intern. pour l'exploration de la mer, 3, 1 (1928).

${ }^{15}$ Driggus, B. F., and Pepper, B. B., J. Econ. Ent., 29, (3), 477 (1936)

$x^{6}$ Driggus, B. F., and O'Neill, W. J., J. Econ. Ent., 31, (2), 221 (1938).

17 Boyce, H. R., 73rd Rep. Ent. Soc., Ont., 1942, 58 (1943).

18 Ripper, W. E., Brit. P. 505853 (1939).

2 Petherbridge, F. R., and Wright, D. W., J. Min. Agric., 45 (2) $140-148$ (1938)

${ }^{20}$ Ullyett, G. G., Sci. Bull., S. Afr. Dept. Agric., No. 178 \{1938\}.

\section{SOLAR PHENOMENA AND GEOMAGNETISM}

\begin{abstract}
A DISCUSSION on "Solar Phenomena and Some Allied Geophysical Effects" was held at a meeting of the Royal Astronomical Society on March 10. The president of the Society, Prof. E. A. Milne, was in the chair, and the discussion was based upon four papers contributed during the recess by the following authors : Mr. H. W. Newton, of the Royal Observatory, Greenwich ("Solar Flares and Magnetic Storms": second paper); Dr. C. W. Allen, of the Solar Observatory, Canberra ("Relation between Magnetic Storms and Solar Activity"); Mr. M. A. Ellison ("Sunspot Prominences : some Comparisons between Limb and Disk Appearances"); and Dr. H. A. Brück, of the Solar Physics Observatory, Cambridge ("On the Distribution of Intensity within the Solar Corona"). Others who spoke during the discussion were Prof. H. H. Plaskett, Prof. W. H. McCrea, Dr. T. G. Cowling and Dr. A. Hunter.
\end{abstract}

\section{Solar Activity and Magnetic Storms}

Opening the discussion, Mr. Newton referred briefly to the findings of his earlier paper, entitled "Solar Flares and Magnetic Storms", recently published by the Society. A solar flare may be described as a small area of the sun's surface which shows the hydrogen line $(\mathrm{H} \alpha)$ in strong emission instead of absorption; many other lines show reversals. but observations are normally made by $\mathrm{H} \alpha$ light with the spectrohelioscope-Hale's instrument, which has contributed more than any other to our knowledge of these phenomena during the last solar cycle. The term 'chromospheric eruption' has been in use hitherto to describe these sudden appearances of bright emission in the sunspot areas, but the term 'flare' seems, in the light of our present knowledge, to be preferable, since the bright emission itself shows no appreciable movement either laterally across the disk or in a vertical direction.

Flares have been observed since 1934 by international co-operation, under the auspices of the International Astronomical Union, and have been classified, chiefly on the basis of area, on a qualitative scale of 1, 2, 3, the last being the largest and most intense. Mr. Newton now selects from these records and from an earlier list by Hale, a total of thirty. seven flares which, by reason of great extent (area comparable with the largest spot-groups), duration or intensity, are judged to be of outstanding importance, and these are designated $3+$.

The collection of data relating to these specially intense flares and their subsequent discussion lead to results which are of the greatest interest to geophysicists. In the first place, it is very significant that, of the thirty-seven flares listed in Newton's table, magnetic storms began within $2 \cdot 0$ days of the flares in no fewer than twenty-seven cases, and two thirds of the associated storms are 'great' storms, having a range at Greenwich in $D \geqslant 1^{\circ}$, or in $H$ or $V \geqslant 300 \gamma$. Since, even at solar maximum, great storms are rare events (about $3 \cdot 2$ per year), the total number of chance coincidences of a flare day occurring within $2 \cdot 0$ days of the commencement day of a great storm can be shown to be just one for the twentyeight flares in the central zone of the disk. Actually seventeen such coincidences were discovered. The time interval (storm begins minus flare first observed) given by the data is $25 \cdot 7 \pm 1 \cdot 5 \mathrm{~h}$; but the largest five of the great storms give $20 \cdot 3 \pm 0 \cdot 9 \mathrm{~h}$, and this difference is considered to be real in view of the probable errors.

The distribution of intense flares over the sun's disk prior to great magnetic storms was next considered, each flare being regarded as a source-point for corpuscular emission. Dividing the flares into 'central zone' $\left(0^{\circ}-45^{\circ}\right)$ and 'outer zone' $\left(45^{\circ}-90^{\circ}\right)$ flares, and analysing the data by the 'superposed epoch method', there emerges a much closer association between the central zone flares and magnetic storms than is the case for those in the outer zone. Furthermore, since the magnetic records are necessarily more complete than the solar flare records, it is possible to extend the available data by working back from the records of great storms and postulating intense flares on the sun's disk about one day earlier to account for them : these flares would most probably be located near the largest or most active spot. group present on the disk at the time. Maunder and others have, of course, shown that there is a statistical relationship between the central zone passage of great spot-groups and the greatest Inagnetic storms. These 'deduced' flares, like the observed flares associated with great storms, are found to favour the central regions of the disk.

The whole body of evidence is consistent with the following general hypothesis: (1) an intense solar flare emits ultra-violet radiation, reaching the ionosphere at the same moment as the $\mathrm{H} \alpha$ emission seen with the spectrohelioscope, and gives rise to the synchronous wireless fade-outand magnetic 'crotchet'; (2) there is a newly formed, cone-shaped corpuscular stream ejected at the time and place of the flare, having a semi-vertical angle as large as $40^{\circ}$, or 
occasionally larger ; (3) the arrival of this corpuscular stream about twenty-six hours later, reaching the earth in a 'head-on' encounter, is the cause of the great magnetic storm with its incidental auroral effects.

The general results of Newton's second paper show that the close relationship between flare and geomagnetic storm is much less definite when the next intensity class (3) of flare is considered. For flares of medium intensity (2) there appears no appreciable connexion between the dual phenomena. Although it seems that a further step has been made in tracing the solar origin of the more intense magnetic storms, Newton has himself emphasized the fact that the smaller magnetic storms which occur during the minimum of the 11-year solar cycle still remain unrelated generally to visible disk markings. The solar origin of these storms is, however, strongly sug. gested by their tendency to recur at intervals of a solar synodic rotation, as first shown by Maunder forty years ago.

In Dr. Allen's absence, his paper was summarized by Dr. H. A. Brück. Allen's work provides independent confirmation of Newton's conclusions and the well-established association between great magnetic storms and solar flares. Its main importance appears, however, to lie in the attempt to study the cause of those smaller magnetic storms and disturbances the solar origin of which is betrayed by their 27-day recurrence tendency, but which do not show any correlation with observable solar phenomena. Grouping magnetic disturbances observed over a period of thirty-six years according to their recurrence tendency, Allen seeks correlations between the occurrence of the different types of disturb. ance and the observation of large sunspot groups. For a shorter period of about four years he has also investigated possible correlations between magnetic disturbances grouped according to their recurrence tendency, intensity and sudden commencement and the observation of solar flares or radio fade-outs produced by such flares.

The results of his investigation lead Allen to the conclusion that the influence of the $M$-regions on the sun (in which the origin of the minor magnetic disturbances is to be sought) is affected by the presence of sunspots in their neighbourhood. When these are within $40^{\circ}$ of the $M$-region, they seem to deflect the emitted particles in very much the same way in which streamers in the solar corona are deflected by regions around sunspots. This, and the particular persistence of the $M$-regions or recurrent disturbances one or two years before sunspot minimum when the equatorial coronal streamers have their greatest extension, suggests according to Allen that $M$-regions are identical with coronal streamers. Their base appears to cover an extended region on the sun from which the corpuscular emission is constrained to move in limited streams by forces in the solar atmosphere. Allen's $M$-regions are therefore rather different from the relatively small $C$-regions of abnormal emission in the coronal line $\lambda 5303$ found by Waldmeier (1942) and identified by him with the $\boldsymbol{M}$-regions. Further studies of the recurrent disturbances during the present solar minimum might possibly bring a decision in favour of one or other of the two hypotheses.

\section{Prominence Motions}

Describing his paper on sunspot prominences, Mr. Ellison remarked that, hitherto, our knowledge of the forms and motions of prominences had been drawn almost exclusively from the study of elevation pictures taken at the sun's limb. The principal instruments in use have been the visual eye-end spectroscope, the spectroheliograph, and, within the last decade, the Lyot coronagraph and the interference polarizing monochromator. The monochromator, as developed by Pettit at Mount Wilson Observatory, following up the original suggestions of Öhman, promises to prove the most generally useful where the study of rapid motions in prominence features is concerned.

With the development of the spectrohelioscope by Hale in 1926, there arose the possibility of studying the motions of sunspot prominences in plan view upon the sun's surface by light of the $H \alpha$ line. It was immediately found that direct readings of the lineof-sight velocity of prominence streamers could be obtained, through the Doppler shifts of the spectral lines, while the horizontal movements were being followed. Thus arose the three-dimensional method of attack. The disk appearances, when these prominences are to be seen by absorption against the brighter background of the chromosphere, also provide fuller and more continuous records, with special reference to the state of activity and stage of development of the parent spot-group. The precise location of the prominence filaments and arches in relation to the spots is likewise of considerable importance in any discussion of the causes of their rapid movements. Observations of this kind made during the past 11-year cycle of solar activity have been contributed mainly by the observatories at Greenwich, Zurich and Sherborne, and Mr. Ellison explained that the present paper was intended to summarize these findings and to provide a correlation with the corresponding types of limb prominence.

Most frequent in occurrence are the inflowing filaments of short life which originate in the surrounding chromosphere or in 'coronal clouds' formed above the spot area, move along curved trajectories and finally terminate near the boundary of the penumbra with a mean velocity of inflow of $48 \mathrm{~km}$. $/ \mathrm{sec}$. Their mean projected length is $61,000 \mathrm{~km}$. and their movements are found to be independent of the magnetic polarity of the attracting sunspots. These filaments are now regarded as being identical with the 'Fleckenkronen', described by Fényi (1891) from limb observations, and with the "Jets and Rockets" of Evershed's memoir of 1917 : they are therefore placed under Type III $a$ of the Pettit classification (1943).

Complex formations of loops and arches over sunspots are rare, but single and double arches are frequently seen, with the spectrohelioscope, connecting adjacent sunspots of the same group. The direction of motion of the gases is upwards on one side and downwards on the other side of the arch, only one case in thirty-one having been observed where matter was clearly in descent on both sides of the same arch. Because of their Doppler displacements, such objects cannot be recorded in their entirety with the spectroheliograph. The mean distance between the legs of the arches is $43,000 \mathrm{~km}$., and the mean velocities of inflow and outflow are found to be $39 \mathrm{~km}$./sec. and $28 \mathrm{~km}$. $/ \mathrm{sec}$. respectively. Such prominences are identified with the arches of Type IIIb in Pettit's limb classification, and the directions of motion are again independent of the magnetic polarities of the associated sunspots.

A further type of great interest is the flanking prominence (Type IIIc), often of great dimensions, 
the form, growth and decay of which is intimately connected with the life-history of the adjacent spotgroup. These exhibit much internal activity, as well as forming external streamers which leave the tops of the prominences to flow horizontally inwards and downwards into the spot area.

Mr. Ellison emphasized that the slender trajectories, perhaps $100,000 \mathrm{~km}$. in length and no more than $5,000 \mathrm{~km}$. thick, have not received any satisfactory explanation or likely parallel in terrestrial physies. They give one the remarkable impression of being semi-permanent conducting paths along which bright condensations follow one another at frequent intervals over a period of several hours. Such motions were first studied in the cinematograph films taken by McMath. In the horizontal trajectories it would seem that gravity must be compensated by radiation pressure, and that the motive force along the trajectory may be of an electrostatic nature. This, however, introduces a difficulty, for the highly conducting properties of the hot solar gases would appear to prevent the collection of electrostatic charges.

\section{Photometry of the Corona}

Dealing with his investigation of the intensity distribution within the inner corona, Dr. Brück mentioned that Waldmeier had recently published a paper on the same subject in which he found signs of a connexion between intensity gradients and the structure of the corona. Dr. Brück's photometric work confirms Waldmeier's suggestions in greater detail. It has been based upon a plate which was obtained by the late Prof. H. F. Newall during the eclipse of August 30, 1905, in Algiers, and at the same time as the plate from which Waldmeier's conclusions had been derived.

The distribution of intensity has been determined from records made with a microphotometer along seventy-two solar radii equally spaced in position angle, and the results have been represented by a series of isophotes covering an intensity ratio of mag. $2 \cdot 5$. These are sensibly circular, as is to be expected for an eclipse near sunspot maximum, but show considerable local variations corresponding to the structure of the corona on the original negative. Intensity gradients, when derived for a region extending from a distance of about $2^{\prime}$ to a distance of about $5^{\prime}$ from the solar limb, show systematic variations with position angle in the sense that intensities, and therewith densities of scattering electrons, seem to decrease less rapidly with increasing distance from the sun when measured along rays than in 'normal' regions in between. The opposite effect has been observed by von Klüber for streamers in the outer corona, which extend from about $6^{\prime}$ to about $70^{\prime}$ from the limb.

As a consequence of his own work, Waldmeier believes that the inner corona is composed of a spherical shell of electrons, atoms and ions. The electrons, by scattering light from the photosphere, give rise to the continuous spectrum of the corona. Over the sunspot zones, where the ultra-violet and corpuscular radiations are assumed to be most intense, the atoms are ionized to a very high degree, losing as many as fifteen electrons, and are left in metastable states from which emission of the forbidden coronal lines takes place in accordance with Edlén's remarkable hypothesis. The increased ionization leads in turn to a greater electron density, with the consequent brightening of the continuous spectrum which has been observed in these regions.

\section{KILIMANJARO: AN ACTIVE VOLCANO}

\author{
By Dr. P. E. KENT
}

$I^{1}$ $T$ is nearly a century since Kilimanjaro was dis covered, a snow-capped mountain 200 miles south of the equator, and the highest point in Africa. Until recently it was thought to be quite extinct, but ex. peditions during the last few years have produced evidence of a recrudescence of activity in the broad Kibo crater which forms the more easterly of the twin summits.

Meyer ${ }^{1}$, the first explorer to climb the mountain, observed that in 1889 the crater floor was almost entirely covered with weathered ice, commenting "the volcanic activity of Kilimanjaro is now a thing of the past; there is no trace even of fumaroles". He recognized, however, that the local emergence of the rock floor indicated a relic of internal heat.

From the time of the first discovery, however, the amount of ice has been diminishing. In 1889 the crater basin discharged ice through a gap in the western side, but in 1906 discharge had ceased, and there has been no connexion between the crater ice and the glaciers of the mountain slopes since that time. A detailed map by Klute ${ }^{2}$ dating from 1912 showed large isolated masses of ice and snow on the crater floor, including a mass practically in the centre, and in 1921 Gillman $^{3}$ was able to detail considerable reductions in the ice masses, which he thought might be due to climatic fluctuation. In the next few years further evidence of the reduction was obtained ${ }^{4}$, and Mittelholzer ${ }^{5}$ published a series of air photographs taken early in 1928 which showed the crater floor mantled with only a thin and patchy covering of snow, and showed a perfectly preserved central crater pit (located where Klute had mapped an "Eisburg") which had apparently not been seen before. Subsequent air photographs show that snow conditions were virtually unchanged in 1932 , but that there was a temporary increase in quantity in $1937^{\circ}$ and that in mid-1942 the greater part of the rock floor was clear ${ }^{7}$. There was thus a rapid decrease in the snow cap in the period preceding 1928, fluctuation during 1928-37, and subsequent further reduction.

H. W. Tilman ${ }^{8}$ climbed the mountain in 1930 and 1933. He found the snow on the outer slopes much reduced between the two visits, and in 1933 observed sulphurous fumes and pieces of sulphur in the crater - the first signs of the gas emission which has since greatly increased.

Two years ago, fresh evidence was obtained. J. J. Richard ascended the mountain in the autumn of 1942 and saw several well-developed fumaroles surrounded by sulphur deposits, and found that the rocks were warm in several places ${ }^{2}$. Early in 1943, he climbed the mountain again in company with $\mathrm{Mr}$. Spink of the Meteorological Department of East Africa $^{10}$, and found that the number of fumaroles had increased from about six to twenty, and that sulphurous fumes could be detected at some distance. Reports from the westerm side of the mountain described underground rumbling noises and earth tremors.

Spink $^{11}$ has since reported a visit to the crater on July 18, 1943, and a detailed account of the phe- 